\title{
NEAR-BAND EDGE SPECTRAL HOLE IN QUANTUM WELL: NO EVIDENCE FOR SUBPICOSECOND PLASMA THERMALIZATION
}

\author{
M. MošKo AND A. MošKová \\ Institute of Electrical Engineering, Slovak Academy of Sciences \\ Dúbravská cesta 9, 84239 Bratislava, Slovak Republic
}

\begin{abstract}
We show that at low carrier energies and densities the carriers in a two-dimensional Coulomb gas interact via classical unscreened carriercarrier collisions. This allows-us to calculate exactly the thermalization due to the two-dimensional carrier-carrier collisions in a nonthermal low-density $\left(\approx 10^{10} \mathrm{~cm}^{-2}\right.$ ) two-dimensional plasma excited near the band edge of an undoped GaAs quantum well. The thermalization is found to be 10-15 times slower than the $200 \mathrm{fs}$ thermalization deduced from the previous spectral-hole burning measurements, which means that the spectral hole does not reflect the thermalization process. We also show that the Born approximation fails in describing such carrier-carrier collisions.
\end{abstract}

PACS numbers: 73.50.-h, 73.50.Bk, 78.47.+p

A nonthermal two-dimensional (2D) electron-hole (e-h) plasma can be excited in a quantum well (QW) using a quasi-monoenergetic laser pulse of shorter duration than the plasma thermalization time [1-3]. Immediately after the excitation the differential transmission (DT) $D T=N_{\mathrm{w}} L_{\mathrm{w}}\left[\alpha_{0}(\omega)-\alpha(\omega, t)\right]$ can be probed by a short broad-band pulse. Here $\alpha_{0}(\alpha)$ is the absorption of the unexcited (excited) QW, $N_{\mathrm{w}}$ - the number of the QWs, $L_{\mathrm{w}}$ - the QW width, $\hbar \omega$ - the photon energy, and $t-$ the time delay of the probe pulse. Neglecting coherence effects [4] the band-to-band absorption reads [3] $\alpha(\omega, t)=\alpha_{0}(\omega)\left[1-f_{\mathrm{e}}\left(\varepsilon_{\mathrm{e}, \mathrm{t}}\right)-f_{\mathrm{h}}\left(\varepsilon_{\mathrm{h}, \mathrm{t}}\right)\right]$, where $f_{\mathrm{e}, \mathrm{h}}$ is the carrier distribution, $\varepsilon_{\mathrm{e}}+\varepsilon_{\mathrm{h}}=\hbar \omega-E_{\mathrm{g}}, \varepsilon_{\mathrm{e}, \mathrm{h}}=\hbar^{2} k^{2} /\left(2 m_{\mathrm{e}, \mathrm{h}}\right)$ is the carrier energy, $m_{\mathrm{e}, \mathrm{h}}$ the effective mass, and $E_{\mathrm{g}}$ the effective band gap of the QW. Then $D T \propto f_{\mathrm{e}}\left(\varepsilon_{\mathrm{e}, \mathrm{t}}\right)+f_{\mathrm{h}}\left(\varepsilon_{\mathrm{h}, \mathrm{t}}\right)$ and this simple DT model is believed [1-3] to provide experimentally the thermalization of $f_{\mathrm{e}}+f_{\mathrm{h}}$. The 200 fs thermalization was observed in undoped GaAs QWs $[1,2]$ for the excited e-h pair density $N_{\mathrm{s}} \approx 10^{10} \mathrm{~cm}^{-2}$ and ascribed to $2 \mathrm{D}$ carrier-carrier (c-c) scattering assuming the carrier excitation below the optic phonon emission threshold. However, this interpretation has no support in semiclassical Monte Carlo (MC) simulations [5] and molecular dynamics (MD) simulations [5], which give a much slower thermalization. The model $D T \propto f_{\mathrm{e}}+f_{\mathrm{h}}$ should be tested by comparing the experimental DT 
with an exact calculation of the thermalization of $f_{\mathrm{e}}+f_{\mathrm{h}}$. In this paper we succeed in performing such calculation for the low-energy low-density 2D plasma $[1,2]$, since (as we show) the carriers in such plasma interact via classical unscreened 2D c-c collisions.

In Refs. [1,2] electrons and heavy holes were excited into the lowest QW subband. Let us consider classical binary collisions in such plasma. A carrier $\gamma$ changes its wave vector from $k$ to $k^{\prime}$ by a collision with a carrier $\delta$, which is scattered from $k_{0}$ to $k_{0}^{\prime}$. The angle $\Theta$ between the initial relative wave vector, $\boldsymbol{g}=\mu\left(k_{0} / m_{\delta}-k / m_{\gamma}\right)$, and the final one, $g^{\prime}$, is [6]

$$
\Theta=\pi-2 \int_{r_{\min }}^{\infty} \mathrm{d} r \frac{b}{r^{2}\left[1-(b / r)^{2}-4 V(r) / \mu g\right]^{1 / 2}}, \quad \gamma=\mathrm{e}, \mathrm{h}, \quad \delta=\mathrm{e}, \mathrm{h},
$$

where $b$ is the impact distance, $r-$ the in-plane distance between the carriers, $r_{\min }$ - the minimum in-plane distance, $\mu=2 m_{\gamma} m_{\delta} /\left(m_{\gamma}+m_{\delta}\right)$, and

$$
V(r)=\frac{1}{(2 \pi)^{2}} \int \mathrm{d} Q \mathrm{e}^{\mathrm{i} Q \cdot r} \frac{q_{\gamma} q_{\delta}}{2 K Q} \frac{F_{1111}(Q)}{\varepsilon(Q)}
$$

where $q_{\mathrm{e}}=-e, q_{\mathrm{h}}=e, K$ is the material permittivity, $F_{1111}(Q)-$ the form factor [5] and $\varepsilon(Q)$ - the "quasi-dynamic" screening function [5]. For unscreened interáction $(\varepsilon(Q)=1)$ between strictly 2D carriers $\left(F_{1111}(Q)=1\right)$ one has $V(r)=$ $q_{\gamma} q_{\delta} / 4 \pi K r$, Eq. (1) gives Rutherford formula $b=(2 G / g) \cot (\Theta / 2)$ where $G=$ $\mu e^{2} / 4 \pi K g \hbar^{2}$, and equation $\sigma_{\mathrm{cl}}=-\mathrm{d} b / \mathrm{d} \Theta$ gives the differential cross-section $\sigma_{\mathrm{cl}}=$ $G /\left[g \sin ^{2}(\Theta / 2)\right]$. The same $\sigma_{\mathrm{cl}}$ follows from the exact quantum cross-section $\sigma=$ $G \tanh (\pi G) /\left[g \sin ^{2}(\Theta / 2)\right][5]$ for $\pi G \gg 1$, while for $\pi G \ll 1$ one gets the Born approximation $\sigma_{\mathrm{B}}=\pi G^{2} /\left[g \sin ^{2}(\Theta / 2)\right]$. Figure 1 shows $\sigma_{\mathrm{B}} / \sigma$ and $\sigma_{\mathrm{cl}} / \sigma$ versus $g$ for the e-e, e-h, and $\mathrm{h}-\mathrm{h}$ collisions. When $g$ is small, $\sigma_{\mathrm{B}} \gg \sigma$ and $\sigma_{\mathrm{cl}}=\sigma$. Thus,

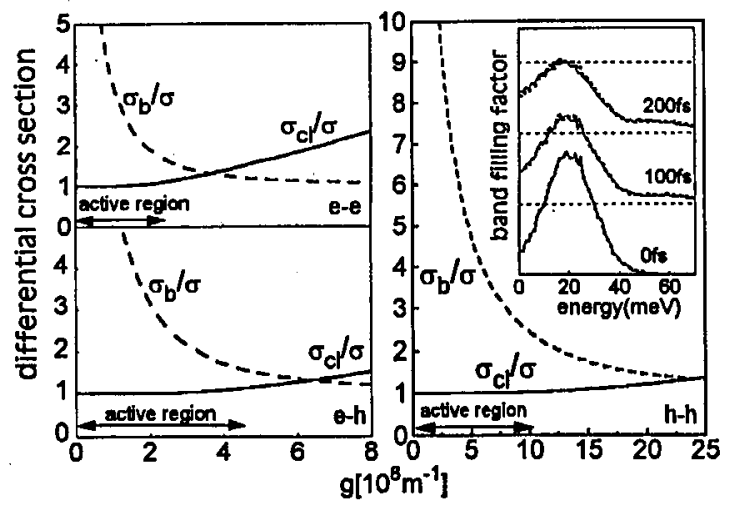

Fig. 1. Unscreened differential cross-section versus $g$ for the $e-e, e-h$, and $h-h$ collisions. The classical cross-section $\sigma_{\mathrm{cl}}$ and the Born cross-section $\sigma_{\mathrm{B}}$ are compared with the exact quantum cross-section $\sigma$. The inset shows the thermalization of the band filling factor $f_{\mathrm{e}}+f_{\mathrm{h}}$ (see the text) for classical c-c collisions with $\dot{g}$-values restricted to the "active region" (dotted lines) and with $g$-values without any restriction (full lines). 
if the many-body Coulomb interaction behaves like unscreened classical binary collisions with cross sections $\sigma_{\mathrm{cl}}=\sigma$, quantum collisions can be simulated exactly by simulating (via the $\mathrm{MC}$ simulation) classical collisions. The collision rate of the carrier $\gamma$, colliding with carrier $\delta$, reads

$$
\Gamma_{\gamma-\delta}(k)=\sum_{k_{0}, s_{0}} f_{\delta}\left(k_{0}\right) \frac{\hbar g}{\mu} 2 b(\Theta=0, g), \quad \gamma=\mathrm{e}, \mathrm{h}, \quad \delta=\mathrm{e}, \mathrm{h},
$$

where $2 b(\Theta=0, g)$ is the total collisional cross section and $s_{0}$ - the spin of carrier $\delta$. In the MC simulation each carrier in the e-h plasma undergoes stochastic binary collisions according to the collision rates (3). The impact distance $b$ realized in a binary collision of two carriers of wave vector $g$ is selected at random between 0 and $b(\Theta=0, g)$ and the scattering angle $\Theta=\Theta(b, g)$ is obtained from Eq. (1). This classical MC simulation is similar to the semiclassical one [5], except that the classical rate (3) diverges because Eq. (1) gives $b(\Theta=0, g) \rightarrow \infty$ even for screened $V(r)$. Therefore we replace $b(\Theta=0, g)$ by a constant finite maximum impact $b_{\max }$, apply the usual MC algorithm [5], and verify that the results do not depend on the choice of $b_{\max }$ for large enough $b_{\max }$. We first excite electrons and holes of density $N_{\mathrm{s}}=2 \times 10^{10} \mathrm{~cm}^{-2}$ instantaneously into Gaussian energy distributions of widths $17.4 \mathrm{meV}$ and $2.6 \mathrm{meV}$, centered at energies $17.4 \mathrm{meV}$ and $2.6 \mathrm{meV}$, respectively $[1,3]$. The $100 \mathrm{fs}$ duration of the excitation [1] is considered later on. We consider the carrier-optic phonon scattering at $300 \mathrm{~K}$ and the $\mathrm{c}-\mathrm{c}$ collisions due to screened interaction (2).

Using $F_{1111}=1$ in Eq. (2) we first discuss the case of $c-c$ collisions between strictly 2D carriers. Our classical MC gives the same plasma thermalization for any $b_{\max }>d$, where $d=\left(2 N_{\mathrm{s}}\right)^{-1 / 2} / 2$ is half of the mean interparticle distance. Figure 2a illustrates this finding for $b_{\max }=d$ and $b_{\max }=1000 d$. Figure $2 \mathrm{a}$ also shows the result obtained for unscreened $c-c$ collisions with $b_{\max }=d$. The result is the same as for screened $\mathrm{c}-\mathrm{c}$ collisions with $b_{\max }=d$ and $b_{\max }=1000 d$, which means that the screening of thermalizing $\mathrm{c}-\mathrm{c}$ collisions (collisions with $b<d$ ) is negligible. This allows us to conclude that our classical MC simulates exact quantum $c-c$ collisions, because the unscreened classical and unscreened exact cross sections are the same. Indeed, the inset to Fig. 1 shows that the classical MC gives the same thermalization when only the $c-c$ collisions for $g$ from the "active region" are taken into account. For this region $\sigma_{\mathrm{cl}}=\sigma$ to a good precision. Figure $2 \mathrm{~b}$ shows the semiclassical $\mathrm{MC}$ result [5], which treats the $\mathrm{c}-\mathrm{c}$ collisions in the Born approximation. The thermalization is fast when compared with the exact c-c collisions treatment in Fig. 2a, which is consistent with Fig. 1, where $\sigma_{\mathrm{B}} \gg \sigma$ in the "active region". Full circles in Fig. $2 \mathrm{~b}$ show the result of the MD simulation [5] of classical many-body Coulomb interactions. The agreement with the MC simulation of classical binary collisions in Fig. 2a proves that the thermalization in the system with many-body Coulomb interactions is governed by unscreened binary collisions with cross sections $\sigma_{\mathrm{cl}}=\sigma$. Finally, our classical MC gives the same result as in Fig. 2a, when a realistic form factor is used in Eq. (2) instead of $F_{1111}=1$. The insensitivity of $\mathrm{c}-\mathrm{c}$ collisions to the form factor is an important condition of validity of the classical description, because $\sigma_{\mathrm{cl}}=\sigma$ (Fig. 1) holds only for $V(r) \propto r^{-1}$. $F_{1111}$ affects $V(r)$ negligibly for $r>L_{\mathrm{w}}=9.6 \mathrm{~nm}$. Since in 


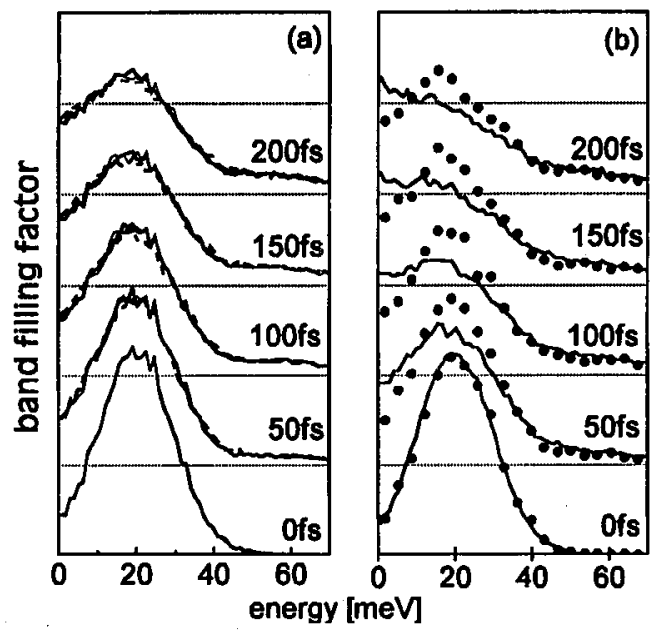

Fig. 2. Band filling factor $f_{\mathrm{e}}+f_{\mathrm{h}}$ versus excess photon energy for times $0, \ldots, 200$ fs. Figure (a) shows the results of the MC simulation of screened classical c-c collisions with $b_{\max }=d$ (full lines) and $b_{\max }=1000 d$ (dashed lines) as well as of unscreened classical c-c collisions with $b_{\max }=d$ (thin full lines). Figure (b) shows the semiclassical MC results [5] (full lines) and the classical MD results [5] (full circles). The spacing between the horizontal grid lines is $\mathbf{0 . 0 3}$.

our simulations $r_{\min }>9.6 \mathrm{~nm}$ in most of the c-c collisions, the effect of $F_{1111}$ on scattering angles (1) is negligible.

To compare with experiment [1] we replace the instantaneous excitation by the carrier generation rate $g(k, t)=\int \mathrm{d} \omega I(\omega, t) \alpha(\omega, t) L_{\mathrm{w}} / \omega$, where $I(\omega, t)$ is the Gaussian pump spectrum with the central frequency $\hbar \omega_{0}=1509 \mathrm{meV}$, the FWHM of $20 \mathrm{meV}$, and the time duration of $100 \mathrm{fs}$ [1]. We simulate $D T=N_{\mathrm{w}} L_{\mathrm{w}} \alpha_{0}(\omega)$ $\times\left(f_{\mathrm{e}}+f_{\mathrm{h}}\right)$, where $\alpha_{0}(\omega)=\left(2.7 \times 10^{5} \mathrm{~m}^{-1}\right) \times$ Sommerfeld factor [7]. Figure 3a compares the experimental DT [1] with the DT simulated by our classical MC for $N_{\mathrm{s}}=2 \times 10^{10} \mathrm{~cm}^{-2}$ and $N_{\mathrm{s}}=1.1 \times 10^{10} \mathrm{~cm}^{-2}$. The former $N_{\mathrm{s}}$ is the estimation from Ref. [1], but the latter $N_{\mathrm{s}}$ gives a better fit of the spectral hole at $0 \mathrm{fs}$. In both cases the relaxation of the spectral hole is much slower than in the experiment. Figure $3 \mathrm{a}$ also shows that the excess energy $\hbar \omega_{0}-E_{\mathrm{g}}=20 \mathrm{meV}$ (estimation from Refs. [1,3]) is too low, because the heavy-hole exciton position of $1457.5 \mathrm{meV}$ and the exciton binding energy of $10 \mathrm{mel}$ [7] give $E_{\mathrm{g}}=1467.5 \mathrm{meV}$ and $\hbar \omega_{0}-$ $E_{\mathrm{g}}=41.5 \mathrm{meV}$. When $\hbar \omega_{0}-E_{\mathrm{g}}=41.5 \mathrm{me} \cdot \mathrm{V}$, the DT relaxation is faster due to increased optic phonon emission. This is shown in Fig. 3b for $N_{\mathrm{s}}=1.1 \times 10^{10} \mathrm{~cm}^{-2}$ (thick full lines). When only the c-c collisions are taken into account, we obtain 15 times slower thermalization (dashed lines in Fig. 3b). We conclude that in Ref. [1] the 2D c-c collisions are obscured by phonon emission due to high $\hbar \omega_{0}-E_{\mathrm{g}}$. The phonons weakly affect MC results in Fig. 3a. These results are thus more relevant to the experiment of Ref. [2], where $\hbar \omega_{0}-E_{\mathrm{g}} \approx 10 \mathrm{meV}$. The observed spectral-hole relaxation [2] is much faster than the thermalization in Fig. 3a, i.e., the spectral hole does not reflect the thermalization of $f_{\mathrm{e}}+f_{\mathrm{h}}$, but probably only 

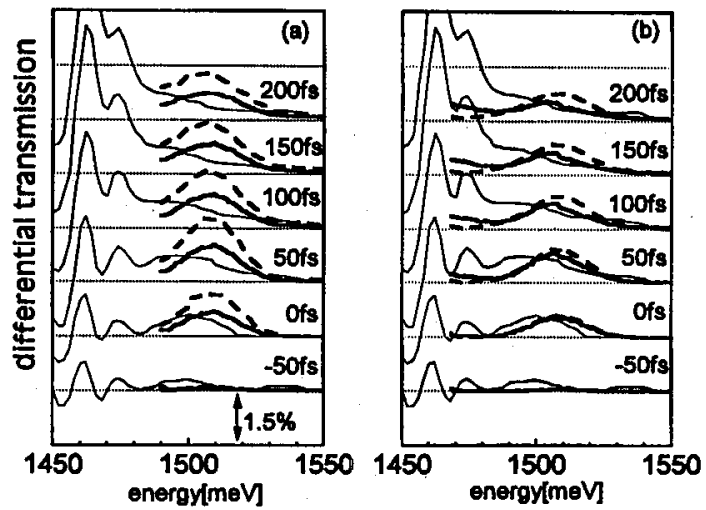

Fig. 3. Differential transmission (DT) versus photon energy. The experimental DT [1] is shown in thin full lines. Figure (a) shows the classical MC results for $N_{s}=1.1 \times 10^{10} \mathrm{~cm}^{-2}$ (full lines) and $N_{\mathrm{s}}=2 \times 10^{10} \mathrm{~cm}^{-2}$ (dashed lines). In these calculations $\hbar \omega_{0}-E_{\mathrm{g}}=$ $20 \mathrm{meV}$. Figure (b) shows the classical MC results for $N_{\mathrm{s}}=1.1 \times 10^{10} \mathrm{~cm}^{-2}$ with optic phonon scattering omitted (dashed lines) and included (full lines). Here $\hbar \omega_{0}-E_{\mathrm{g}}=$ $41.5 \mathrm{meV}$.

coherence effects. Unfortunately, available theories of coherence effects [4] treat the $3 \mathrm{D}$ c-c scattering in the Born approximation, which breaks down in our $2 \mathrm{D}$ case (Figs. 1,2).

\section{References}

[1] W.H. Knox, C. Hirliman, D.A.B. Miller, J. Shah, D.S. Chemla, C.V. Shank, Phys. Rev. Lett. 56, 1191 (1986).

[2] J.B. Stark, W.H. Knox, D.S. Chemla, Phys. Rev. Lett. 68, 3080 (1992).

[3] W.H. Knox, in: Hot Carriers in Semiconductor Nanostructures, Ed. J. Shah, Academic Press, New York 1992, p. 313.

[4] F. Rossi, S. Haas, T. Kuhn, Semicond. Sci. Technol. 9, 411 (1994).

[5] M. Moško, A. Mošková, V. Cambel, Phys. Rev. B 51, 16860 (1995).

[6] R. Balescu, Equilibrium and Nonequilibrium Statistical Mechanics, Wiley, New York 1975.

[7] D.S. Chemla, D.A.B. Miller, P.W. Smith, A.C. Gossard, W. Wiegmann, IEEE J. Quantum Electron. 20, 265 (1984). 